\title{
JRC2006-94037
}

\section{WHEEL WEAR LOSSES FROM BOGIE ROTATION RESISTANCE, EFFECTS OF CANT AND SPEED}

\author{
Scott A. Simson \\ Michelle E. Pearce \\ Centre for Railway Engineering \\ Faculty of Engineering Central Queensland University \\ Rockhampton, Queensland 4702 \\ Australia \\ E-mail s.simson@cqu.edu.au
}

\begin{abstract}
Previous investigation on wheel wear losses from bogie rotation resistance have only considered as designed curve operations [1, 2], with a small cant deficiency present in the curve design. Results in such curves show a relatively small increase in overall wheel and rail wear due to bogie rotation resistance. The bulk of increased wheel/rail wear is caused by rotation friction moments at the centre bearing, which are generated in the curve transition, being retained into the constant radius curve. Cant Deficient design curves are shown to not retain the full rotational friction moment that is generated in curve transitions. The wear losses on individual wheelsets is shown to be greatly affected by bogie rotation resistance even when the total wheel rail wear for the wagon is not greatly altered. The wheel/rail wear is best described by classifying curves by the number of wheelsets in flange contact with the high rail.
\end{abstract}

The Queensland coal operations have proportionally much higher wear rate increases attributed to bogie rotation friction. Large reductions of wheel wear have been anecdotally report by QR rollingstock maintenance staff following the introduction of lubricating polymer centre bearing liners. Case study simulations show that the track's dominant tight curve is very sensitive to high centre bearing friction. The curves occur on a 1:50 grade for which loaded traffic is downhill at $40 \mathrm{kph}$ compared to the empty traffic climbing at the speed limit of 60 $\mathrm{kph}$. Hence these dominant downhill curves have a cant excess.

Bogie rotation friction management is shown to be particularly important on rollingstock with low warp stiffness that operate through tighter curves at lower than the cant design speed. Such rollingstock will exhibit significantly shortened wheel maintenance cycle times due to high bogie rotation friction retained into the curve and high wheelset angles of attack seen on the leading bogie. Wear rates can become further accelerated due to the negative impact high angles of attack have on gauge face lubrication. Bogie warp and wheelset angles of attack retained in constant radius curves are affected by lateral forces such as coupler angling train forces.

\section{INTRODUCTION}

Rail CRC Australia have been investigating the outcomes of Bogie Rotation Friction Management. Wear losses in three piece bogies from high bogie rotation resistance have been calculated using vehicle simulations. Bogie rotation resistance has been modeled accounting for uneven loading effects in track curve transitions [3]. The vehicle model accounts for the freeing of friction restraints to bogie warp with up and down movement of the bogie's secondary suspension. The simulations have been performed using traditional three piece super-service bogies both with and without steering arms' gap and constant contact side bearers. Additional simulations have made a case study of 106 tonne coal wagon operations in Central Queensland where rollingstock operations are unidirectional due to the bottom drop door operation. The case study wagon chosen was anecdotally reported to the Rail CRC project steering committee as having large reductions in wheel wear following the introduction of polymer liners.

\section{VEHICLE DYNAMICS SIMULATION}

The vehicle dynamics simulation work has been conducted using the software package VAMPIRE ${ }^{\circledR}$. The vehicle model itself has been specially formulated to model the bogie rotational forces in transition curves [3] and interaction involved in bogie warp. Four key connections need to be modeled with non-linear spring stiffness and friction damping to portray the warp and bogie rotation effects. These connections are the center bowl top center, the constant contact 
side bearer (CCSB), the bearing adaptor and the friction wedge connections.

The high complexity from modeling such a high number of non-linear elements necessitates a high computational load and small step size. To improve the vehicle model performance some linear elements have been included in some connections to allow a courser step size. Most of the simulations have been performed with a step size frequency of $20-50 \mathrm{KHz}$. For some of the simulation runs in very tight curves there have been numerical instability problems.

\subsection{Bogie Connection Models}

The center bowl / top center connection is modeled with six vertical non-linear springs arranged to handle the narrow gauge $(1067 \mathrm{~mm})$ top center design (Figure 1) used in QR National operations. The model is focused on friction effects and ignores the damping included in Katta and Conroy's modeling [4]. The six non-linear springs are linked to planar friction elements to account for the effects of longitudinal and lateral movements on the rotational friction. Eight non-linear springs model the center bowl rim and also have matching planar friction elements to model rim frictions and handle bolster pitching forces in longitudinal impacts [5].

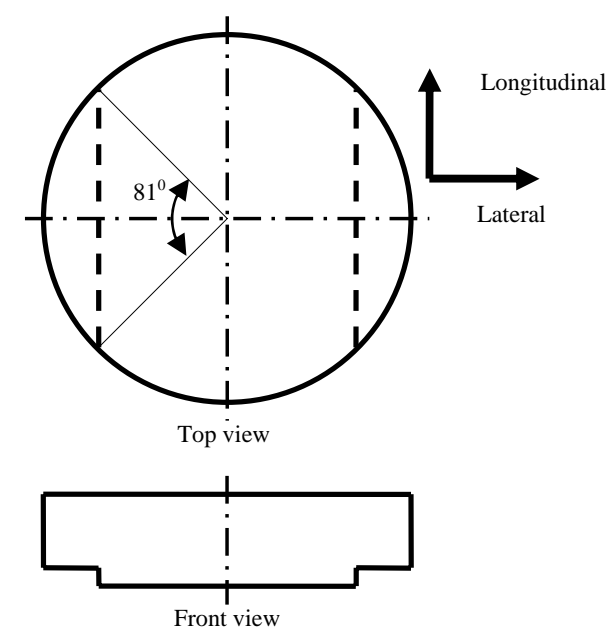

Figure 1 Narrow Gauge Top Center

The CCSB model is based of a Stucki roller assisted normal travel side bearer design. The preload on the wear pads of CCSB is around $70 \%$ of the tare mass of the wagon body. Some simulations were performed with a gap type side bearer modeled.

The friction wedge model is a simplified friction element with a variable friction coefficient for the up and down movements of the wedge. The model used is a ride control bogie design. The wedges are modeled with planar friction to link the warping and bouncing interactions at the secondary suspension. Due to shortcomings in the VAMPIRE® package the lateral frictions coefficients are inaccurate, but this is of small consequence compared to linking the bounce and warp resistances provided by the friction wedge.
The bearing adaptor is modeled with twin non-linear springs for the vertical load and nonlinear springs for lateral and longitudinal movement. Twin planar friction elements are used to model the lateral, longitudinal and rotational friction forces at the adaptor.

Steering arm bogies were used in some of the simulations to compare bogie type performances. The steering arm connection was modeled with a simple single axis linear spring connection made laterally between wheelsets.

\section{CURVING WHEEL WEAR RESULTS}

Curving performance simulations were done for a variety of bogie types over a range of curvatures and occasionally with a range of cant or speed combinations to give variable cant deficiencies. Performance was assessed on a wear index basis where the wear index is representative of wear energy being the wheel rail creep forces multiplied be the wheel rail creepages. Contact patch spin creep is ignored.

\subsection{Curving Behaviour by Bogie Type}

Curving behavior due to centre bowl friction was found to be dependent on the curve radius and wheel profiles. Much of the curving behavior could be classified based on the numbers of wheelsets in flange contact with the high rail. Behavior of steering arm bogies and non-steering arm bogies are very different due to the warp stiffeness provided by the steering arm.

Five curve types can be identified by the numbers of wheels in flange contact. The five types are:

Tight Curves: where all four wheelsets flange against the high rail. Bogie rotational friction has little impact on total wear but does increase the wear on the front bogie compared to the rear bogie.

Near Tight Curves: where three wheels are in contact, wheelsets one two and three. Near tight curves occur only with bogies having low warp stiffness. Wear rates increase dramatically with centre bearing friction. Wear rates get heavily wieghted to the front bogie as wheelset one and two have increasing flange contact and angle of attack, but only wheelset three on the rear bogie has reductions in flanging and angle of attack.

Medium Curves: where two of four wheelset flange against the high rail, wheelsets one and three. Bogie rotational friction has a moderate impact on total wear depending on the warp stiffness. Individually wear increases on wheelset one and is reduced partially on wheelset three.

Gentle Curves: one wheel in flange contact being wheelset one. Bogie rotational friction has a high impact on total wear and specifically on the front wheel. However total wear is very small in gentle curves and has little impact on wheel life.

Tangent Curves: no wheelsets are in flange contact. Bogie rotational friction alters the tracking positions, however wear effects are minimal.

Steering arm bogies minimize the effects of bogie rotational frictions. Bogie warping forces are applied to steering the wheelsets, and the wheelset angle of attack is minimized 
reducing any wear increases to a minimum. Only the gentle curve and tangent curve wheelset tracking effects remain significant for the steering arm bogie performance.

\subsection{Cant Deficient Design Curve Performance}

Wheel wear losses generated by high bogie rotational friction is dependent on the amount of the rotational friction moment that is retained from the curve transition when the bogie is made to rotate to the constant radius section of the curve where the majority of wheel wear occurs. This retention of bogie rotation friction is dependent upon multiple factors such as the bogie warp stiffness, curve cant and curve speed, the dynamic movement of the bogies' secondary suspension being a key factor in allowing the un-warping of the bogie and the relief of bogie rotation friction moments. Figure 2 shows how increased track cant alters the retained bogie rotation friction due to increased movement required at the secondary suspension.

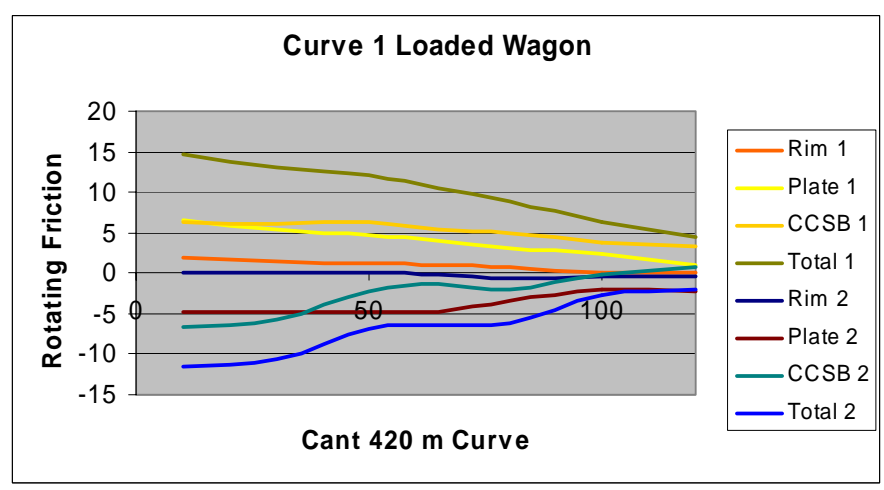

Figure 2 Bogie rotation friction moment retained in constant curve.

\subsection{Curving Wear Performance from Bogie Rotational Friction}

Wheel/Rail wear results from bogie rotation friction increase are given in Figure 3 using a non steering arm bogie.

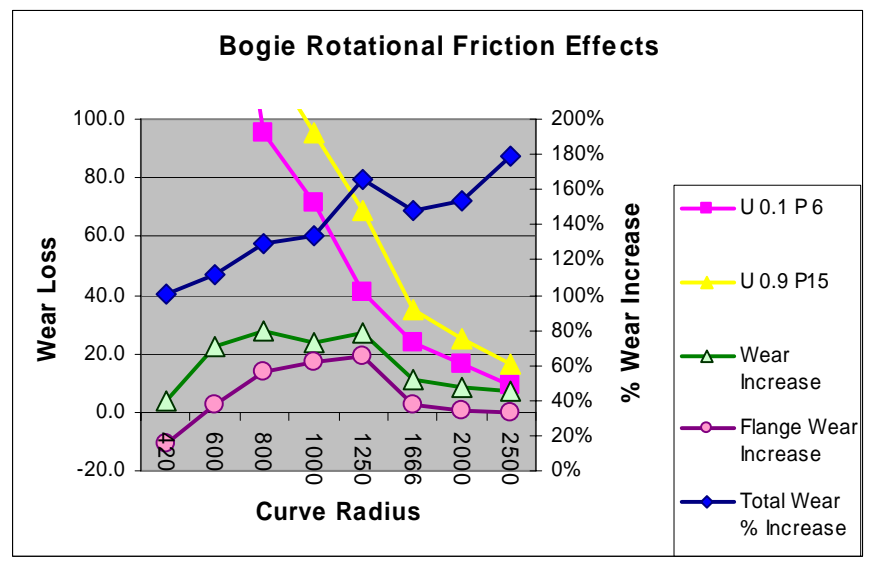

Figure 3 Wear rate increases from bogie rotation friction in cant deficient curves
The data in Figure 3 is only for constant curving and does not include transition curve wear. The bogie model used for these results had high suspension stiffness and warp stiffness. Wear rate increases in this case are limited to moderate and gentle curves, with the most significant losses generated being for the $1250 \mathrm{~m}$ curve radius, which is the highest radius gentle curve where bogie rotation friction produces extra flanging wear on wheelset one.

The distribution of wheel wear across four wheelsets is shown in Figure 4 for medium, gentle and tangent curve types. For wagons with low centre bearing friction the wheel wears are evenly distributed between the front and rear bogie. For the wagons with high centre bearing friction the wheel wear gets increasingly directed to the leading wheelset as the curve radius increases.

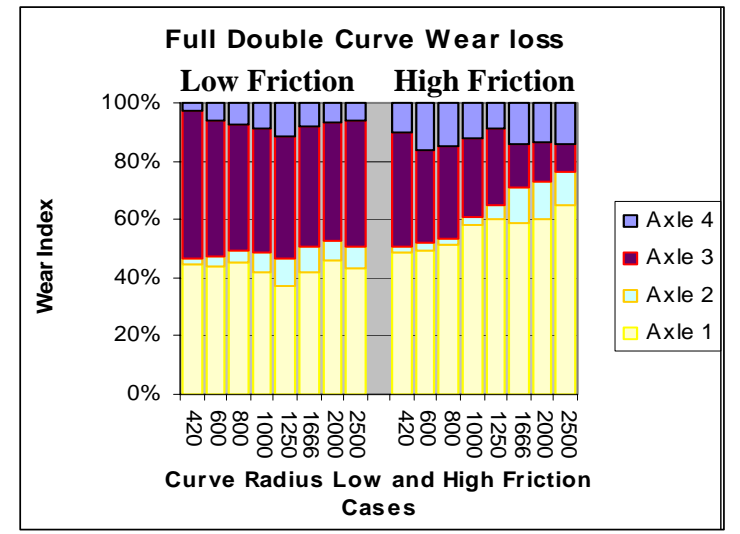

Figure 4 Distribution of wheel wears for low and high friction center bearings

\section{Case Study Curve Performance}

A case study wear analysis was performed in the Goonyella system of QR National in Central Queensland. The bogie from the VSA coal wagon used in the case study is a normal three piece bogie with roller assisted CCSB. The suspension stiffness is lower than that used for the results in Figure 3. Wheel/Rail wear impacts in curving are considerably higher. Table 1 gives the total wear energy impacts across the system assuming no longitudinal train loads. Wear rates increase dramatically as the centre bearing rotation friction increases. Figure 5 shows the wear rate increase for the nine test case curves used in the case study. The case study also included track sections for balloon loops and turnouts but those wear losses were insignificant with $86 \%$ of wheel/rail wear losses occurring in just three most prevalent curve types they being $304 \mathrm{~m}, 500 \mathrm{~m}$ and $808 \mathrm{~m}$ curves.

Table 1 Total wheel/rail wear energy on all curves

Center Bearing $\quad$ New Wheel 1 Worn Wheel

\begin{tabular}{lll}
\hline Friction Coefficient & {$[\mathrm{MJ} /$ wagon $]$} & {$[\mathrm{MJ} /$ wagon $]$} \\
\hline
\end{tabular}

\begin{tabular}{|c|c|c|}
\hline 0.10 & 84.8 & 63.5 \\
\hline 0.30 & 96.1 & 64.2 \\
\hline 0.50 & 104.8 & 82.1 \\
\hline 0.99 & 127.7 & 86.5 \\
\hline
\end{tabular}


The wear impacts from bogie rotation for each curve type including transition curves for both empty and loaded wagons are shown in Figure 5. There is a distinct difference in the result of the $304 \mathrm{~m}$ radius curve. This curve was given special treatment as the majority of such curves are on a steep grade with loaded downhill trains operated at a reduced train speed. Note the Figure 5 data ignores the longitudinal train forces that are present on wagons depending on train position when negotiating the $304 \mathrm{~m}$ radius curves. All other curves operate with a 2/3 cant deficiency.

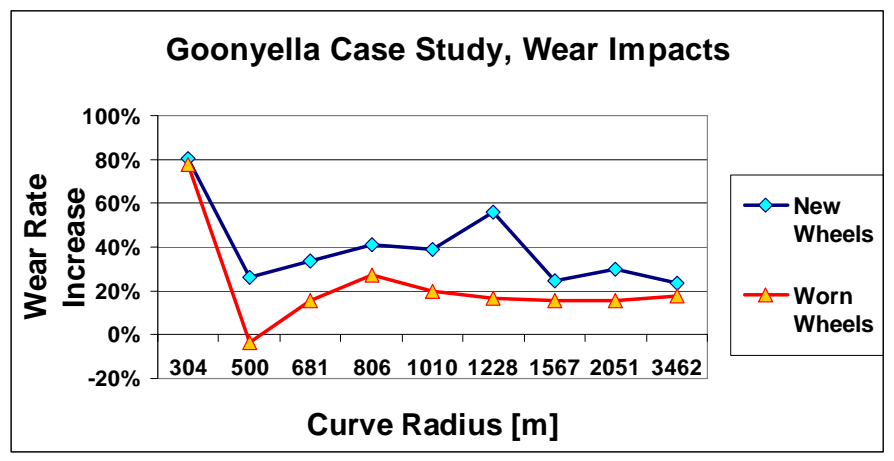

Figure 5 Wear Impacts of Bogie Rotation Friction by
Curves

In Figure 5 ignoring the $304 \mathrm{~m}$ curve we can see that wear rate increase has a peak result of $1228 \mathrm{~m}$ radius curve for the new wheel profile and $808 \mathrm{~m}$ radius curve for the worn wheels. These peak wear rate results are consistent with the Figure 3 peak of $1250 \mathrm{~m}$ curve which represents the last gentle curve; where we define gentle curves as one wheelset in flange contact. The worn wheel profiles having greater effective conicity stop flanging for curve radius greater than the $808 \mathrm{~m}$ radius curve.

The case study simulations of track turnouts showed the greatest increase in wheel/rail wear from bogie rotation friction with wear from both empty and load traffic having a 96\% increase in total wheel/rail wear for damaged centre bowl liner compared to a well lubricated polymer liner. The turnout design was a tangential turnout and thus consisted entirely of transitional curves and no constant curves explaining this high bogie rotational friction sensitivity.

\subsection{Near Tight Curve Performance}

The $304 \mathrm{~m}$ radius curve results in Figure 5 show an increase in the wheel wear for a damaged wear liner surface (coefficient 0.99 ) of $80 \%$ compared to self lubricating polymer (coefficient 0.1 ). Those savings decrease to $40 \%$ for Mn Steel wear liners (friction coefficient 0.5) compared to the well performing polymer liner. Previous simulations (Figure 3, [1]) had suggested that, for such tight curves, wear loss increases with bogie centre bearing friction would be limited to $10-20 \%$. Those previous assessments assume that curving behavior has a direct relationship to curve radius and ignore curve type behavior as related to the number of flanging wheelsets in the one vehicle.
Looking closer at the test case vehicle wear rate increases due to centre bearing friction we see that wear rate increases occur from the loaded wagon case only. In the empty wagon little weight is placed on the centre bowl, hence the friction coefficient has little effect on the total rotation resistance. For the loaded train case, the $304 \mathrm{~m}$ radius curve is a descending grade with steady coupler force under dynamic braking of 540 $\mathrm{KN}$ at the lead locomotives. Behind the lead locomotives the longitudinal forces of the train produce lateral forces against the high rail. The train speed is limited to $40 \mathrm{kph}$ with the track cant designed for $60 \mathrm{kph}$. Hence the curve is cant excessive. The wear results of the loaded train operation are shown for new wheels in Figure 6. Total wheel wear rates for the rear wagon case are increased by $112 \%$, but the front of train wagon wheel wear increases only by $21 \%$.

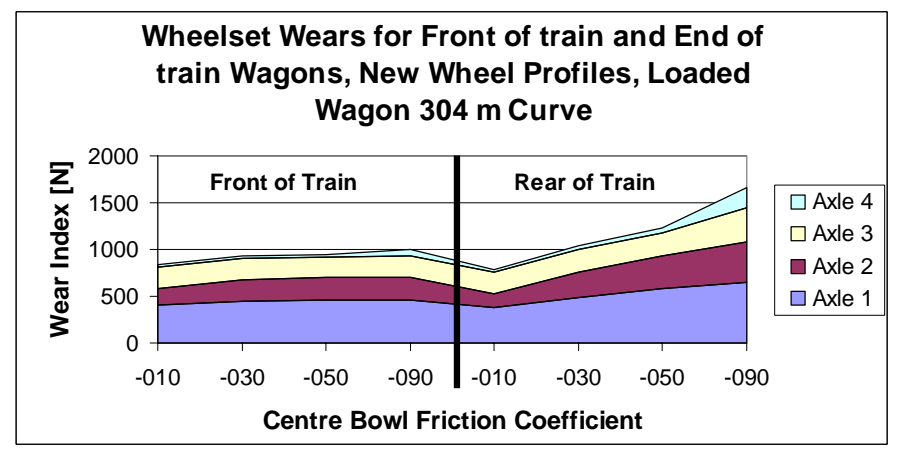

\section{Figure 6 Wheel/rail wear impacts at the front and rear of train using new wheel profiles}

In Figure 6 you can see by the breakdown of wear rates by wheelset position that the fourth axle is the only wheel not flanging. Thus, for the new wheel profile, the $304 \mathrm{~m}$ radius curve is a near tight curve as previously described. In a near tight curve, bogie warp from rotation resistance on the front bogie drives increased wear due to higher angles of attack of the wheelsets. Rear bogie wear reduces but with just one wheel in flange contact that reduction is not nearly as much as the wear increase on the front bogie. The lateral forces at the couplers due to longitudinal train forces and coupler angling in the curve clearly effect the bogie warp and angle of attack generated by the centre bearing rotation friction.

\subsection{Reduced Train Speed Curve Performance}

Further simulations were done on the $304 \mathrm{~m}$ curve to assess the effect of the speed reduction to $40 \mathrm{kph}$ from $60 \mathrm{kph}$. Figure 7 gives the total wheel/rail wear impacts of bogie rotation friction on each wheelset for the two curve speeds.

At $60 \mathrm{kph}$ the wear impacts are significantly reduced, total wear increase being $44 \%$ compared to $112 \%$ seen at $40 \mathrm{kph}$. This is consistent with Figure 5 results with a higher wear increase for near tight curve type at $304 \mathrm{~m}$ radius and higher wear rate increases seen for gentle curve types of 808, 1010 and $1228 \mathrm{~m}$ curve radius. The percentage wear increase is highest for the last gentle curve. However wear rate increases are much greater for the near tight and medium curve types. 
Wear Rate Increase $304 \mathrm{~m}$ Curve at $40 \mathrm{kph}$ and $60 \mathrm{kph}$

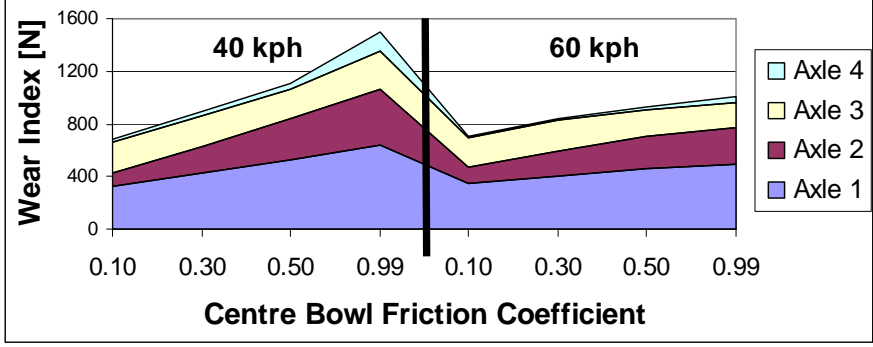

Figure 7 Wheel/rail wear impacts at $40 \mathrm{kph}$ and $60 \mathrm{kph}$

A closer examination of the curving performance differences at $40 \mathrm{kph}$ and $60 \mathrm{kph}$ are given in Figure 8 and Figure 9. In Figure 8 and Figure 9 we see wear index and angle of attack of each wheelset as it negotiates the $304 \mathrm{~m}$ test curve at $40 \mathrm{kph}$ and $60 \mathrm{kph}$ for a wagon with steel on steel wear liners ( 0.5 friction coefficient). In Figure 8 and Figure 9 there is a strong correlation between the wheelset angle of attack and the wheelset wear index. The angles of attack simulated at $40 \mathrm{kph}$ are much higher than those recorded at $60 \mathrm{kph}$. This represents an increase in the bogie warp.

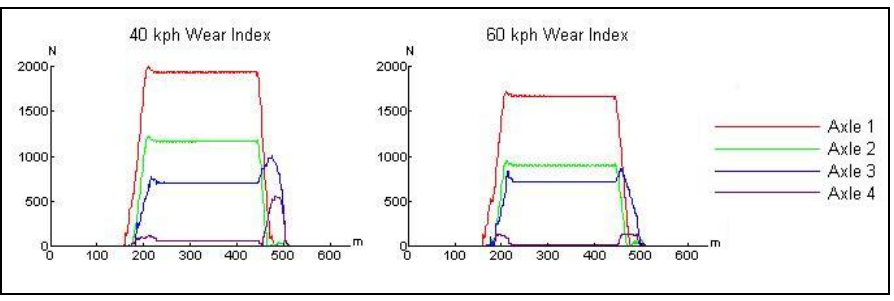

Figure 8 Wheel/rail wear index over $304 \mathrm{~m}$ radius test curve

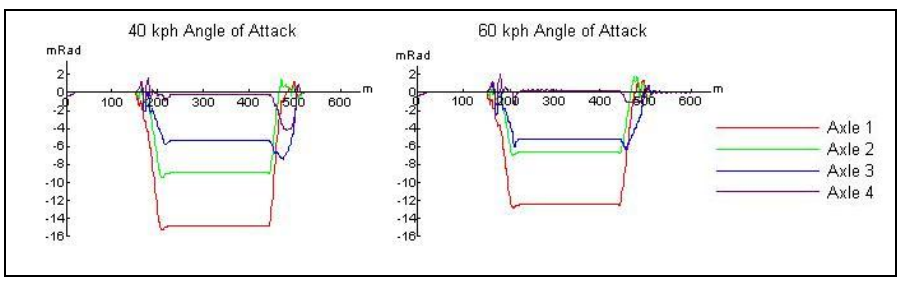

\section{Figure 9 Wheelset angle of attack to the rail over the $304 \mathrm{~m}$ radius curve for steel wear liner ( 0.5 friction coefficient)}

Bogie warp is reversed on the exit transition of the curve and the rear bogie becomes unfavorably warped whilst the lead bogie favorably warps, steering away from the high rail. Figure 9 shows a large angle of attack increase for the rear bogie negotiating the exit curve transition at $40 \mathrm{kph}$ rather than 60 $\mathrm{kph}$. It is assumed from this data that the combination of transition curve shape and vehicle speed allows the VSA vehicle model to warp further at $40 \mathrm{kph}$ then it does at $60 \mathrm{kph}$.

Bogie rotational friction moments (Figure 10) for $40 \mathrm{kph}$ and $60 \mathrm{kph}$ simulations show the level of friction moment retained in the constant curve is greatly reduced for $60 \mathrm{kph}$. The retention of bogie friction moment from transition to curve is regarded as dependent on vehicle movement at the secondary suspension. The greater the vertical movement of each friction wedge the greater any lateral movement can be due to planar friction effects of the wedge.

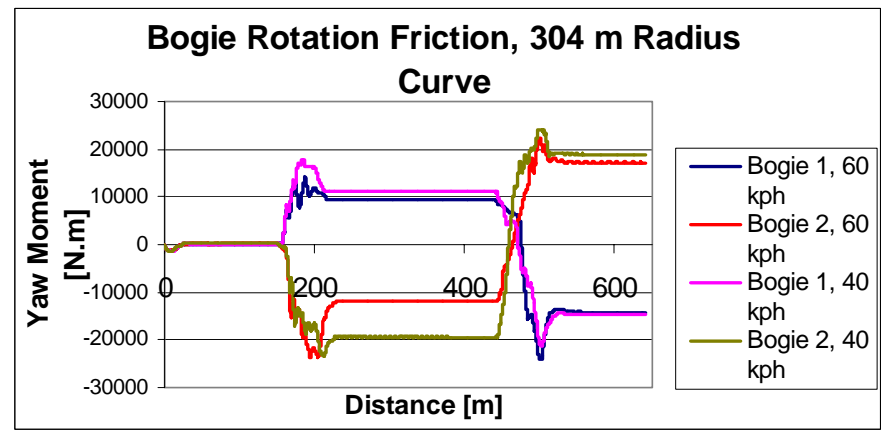

Figure 10 Bogie Rotation Friction in $\mathbf{3 0 4} \mathrm{m}$ radius curve for steel wear liner ( 0.5 friction coefficient)

\subsection{Contact Mechanics, Near Tight Curve}

There are further implications of the high angles of attack that can be generated from the bogie rotation fiction. As angle of attack increases from the neutral or radial position of the curve, the contact position on the flange moves forward from the axle centre line. The direction of creepage across the flange contact patch then becomes downward away from the top of the rail. This has a negative effect on flange/gauge face rail lubrication. Lubricant on the rail is increasingly forced down and away from the gauge face contact band due to the creep movement as angle of attack increases. In the case study $304 \mathrm{~m}$ radius curve (Figure 11), the creepage angle on the flange contact area is increasingly detrimental to lubrication with increases to the center bearing friction coefficient. Lateral rail forces also increase with angle of attack however the increase in contact force is relatively small.

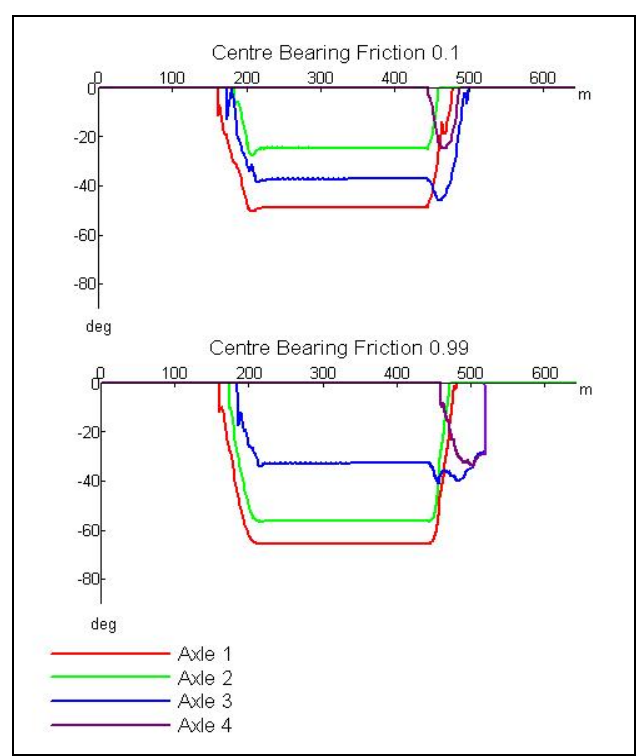

Figure 11 Flange Contact Angle of Creepage, $304 \mathrm{~m}$ Radius Curve 


\subsection{Further Work}

Further work is required to determine the effects of curve transition design, cant and speed in the retention of bogie rotation friction forces from curve transition to constant curving. The present simulation model needs validation against test data for its accuracy in modeling bogie rotation friction forces during transition and resulting bogie warp deflections. Laboratory tests are planned to measure rotation friction forces with track twist. Further simulation testing is planned to investigate differences from side bearer design.

Field testing to validate the simulation is highly desirable though the expense is like to require that simulation data first suggest high prospect of project success and benefit. Further research projects using instrumented vehicles and wayside detection devices are being formulated for new curve transition designs. A project proposal for a renewed Rail CRC involves investigating transition designs to mitigate tight curve wheel squeal using wayside and vehicle mounted monitoring of squeal noise and angle of attack. A specific research test program is being formulated for the case study operations of QR discussed in this paper to monitor wheelset angle of attack using on board and wayside equipment and testing of lubrication conditions. Vehicle instrumentation would also need to measure coupler forces and angles and the train speed.

\section{CONCLUSION}

Bogie rotational modeling needs to account for the planar friction interaction between longitudinal, lateral and yaw movements at the center plate and bearing adaptors. Planar friction interaction between vertical and lateral movements at the friction wedges of the secondary suspension also need to be modeled.

Wagon curving behavior for three piece bogies can be classified into five types relating to the number of wheelsets in flange contact during curving. The curve types being: tight four wheelsets flanging; near tight three wheelsets flanging; medium two wheelsets flanging; gentle curves one wheelset flanging; tangent curves no wheelsets in curve contact. The extent of curve radii that fit the near tight curve classification is dependent on the warp stiffness of the bogie, the lower the warp stiffness the greater the range of curves that could be said to be near tight.

Bogie rotational friction increases wheel rail wear in three piece bogies with lower warp stiffness. The use of steering arms eliminates the most significant effects of bogie rotation friction, reducing wheel/rail wear and angle of attack. Tracking behavior of steering arm bogies in gentle curves and tangent curves is still affected by bogie rotation friction levels.

Wheel/rail wear losses due to centre bearing friction vary depending on the curve type. Tight curve total wear losses are negligibly affected by centre bearing friction however individual wheelsets are greatly affected with a rapid increase in the front bogies wheel wear ahead of reductions in the rear bogies wheel wear. Similarly medium curves experience modest increases in wheel/rail wear rates. Wheel/rail wear rates increase significantly in near tight and gentle curves where the bogie rotation friction directly contributes to an increase in wheel flanging experienced by the leading bogie with little or no wear reduction seen by the trailing bogie. Total wheel wear rate effects become most significant in operations were the dominate tight curve fits a near tight curve classification.

Bogie rotation friction effects are also affected by the curve cant, speed and train forces. Increasing the curve cant and the resultant track twist in transition reduces the retention of bogie rotation friction moments from the curve transition into the constant curvature. Likewise speed, where it causes an increase in the movement at the secondary suspension friction wedges, causes a reduction in the bogie rotation friction moment seen in constant curvature. Lateral forces from train couplers are also shown to affect the retained bogie warp and bogie rotation friction moments generating wheel/rail wear effects in the curve.

\section{ACKNOWLEDGMENTS}

We would like to thank the Cooperative Research Centre for Railway Engineering and Technologies (Rail CRC), Australia for the funding support they have provided to investigate bogie rotation friction management. In particular the Rail CRC industry partner QR are thanked for their support in the provision of case study data.

This research has been conducted utilizing the software VAMPIRE ${ }^{\circledR}$ and the support of AEA Technology Rail.

\section{REFERENCES}

[1] Wu, H., 2002, "Effect of Center Plate Lubrication on Vehicle Curving and Lateral Stability”, Research Report R-959, Association of American Railroads / Transport Technology Center, Inc, Pueblo Colorado.

[2] Wu, H., Robeda, J. Guins, T., 2004, "Truck Center Plate Lubrication Practice Study and Recommendations", Association of American Railroads / Transport Technology Center, Inc. Research Report R-966, Pueblo Colorado.

[3] Simson S. A., Pearce M., 2006, "Centre Bearing Rotation Forces During Curve Transitions”, Proc. Conference on Railway Engineering 2006, April 30 - May 3, RTSA, Melbourne, Preprint

[4] Katta, R. R., Thomas C. F., 2004, “A Dynamic Stiffness and Damping Model for Rail Car Center Plate Polymer Liners” Proc. 2004 ASME/IEEE Joint Rail Conference, ASME, Baltimore Maryland, pp. 63-73

[5] Simson S. A., Pearce M., 2004, "Longitudinal Impact Forces at 3 Piece Bogie Center Bearings" ASME/IEEE Joint Rail Conference, 2005, American Society of Mechanical Engineers, Rail Transportation Division (Publication) RTD, v 29, p 45-50. 